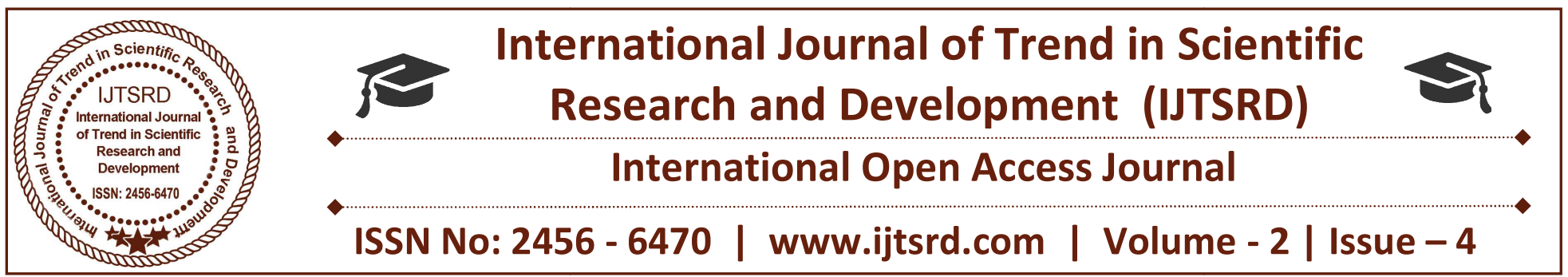

\title{
Artificial Intelligence and Machine Learning's Impact on Market Design
}

\author{
Mrs. Pushpalata S. Patil \\ Lecturer, For Information Technology, \\ Shantiniketan Vidyamandir Sec \& high Sec School [Junior College], Sangli, Maharashtra, India
}

\begin{abstract}
In complex environments, it is challenging to learn enough about the underlying characteristics of transactions so as to design the best institutions to efficiently generate gains from trade. In recent years, Artificial Intelligence has emerged as an important tool that allows market designers to uncover important market fundamentals, and to better predict fluctuations that can cause friction in markets. This paper offers some recent examples of how Artificial Intelligence helps market designers improve the operations of markets, and outlines directions in which it will continue to shape and influence market design.
\end{abstract}

Keywords: ANN; PSO; Fuzzy Logic; Genetic Algorithm.

\section{INTRODUCTION}

For millennia, markets have played a key role in providing individuals and businesses with the opportunity to gain from trade. More often than not, markets require structure and a variety of intuitional support to operate efficiently. For example, auctions have become a commonly used mechanism to generate gains from trade when price discovery is essential. Research in the area now commonly referred to as Market Design.

In complex environments, it can be difficult to understand the underlying characteristics of transactions, and it is challenging to learn enough about them in order to design the best institutions to efficiently generate gains from trade.

But how can market designers learn the characteristics necessary to set optimal, or at least better, reserve prices? Or, more generally, how can market designers better learn the environment of their markets? In response to these challenges, Artificial Intelligence (AI) and Machine Learning are emerging as important tools for market design. Retailers and marketplaces such as eBay, Tao Bao, Amazon, Uber and many others are mining their vast amounts of data to identify patterns that help them create better experiences for their customers and increase the efficiency of their markets.

Turning back to markets for online advertising, twosided markets such as Google, which match advertisers with consumers, are not only using AI to set reserve prices and segment consumers into finer categories for ad targeting, but they also develop AIbased tools to help advertisers bid on ads.

Another important application of AI's strength in improving forecasting to help markets operate more efficiently is in electricity markets. To operate efficiently, electricity market makers such as System Operator must engage in demand and supply forecasting. An inaccurate forecast in the power grid can dramatically affect market outcomes causing high variance in prices, or worse, blackouts. By better predicting demand and supply, market makers can better allocate power generation to the most efficient power sources and maintain a more stable market.

\section{LITERATURE REVIEW}

Imaginative Perception: Labels to Affordances "Although the field of A.I. is exploding with micro discoveries, progress toward the robustness and flexibility of human cognition remains elusive. Not long ago, for example, while sitting with me in a cafe, 
my 3-year-old daughter spontaneously realized that she could climb out of her chair in a new way: backward, by sliding through the gap between the back and the seat of the chair. My daughter had never seen anyone else disembark in quite this way; she invented it on her own and without the benefit of trial and error, or the need for terabytes of labeled data." Gary Marcus, Artificial Intelligence is stuck: Here's how to Move it forward, New York Times Sunday Review, July 29, 2017.

From Data Science to Imagination Science "A theory is not like an airline or bus timetable. We are not interested simply in the accuracy of its predictions.

A theory also serves as a base for thinking. It helps us to understand what is going on by enabling us to organize our thoughts. Faced with a choice between a theory which predicts well but gives us little insight into how the system works and one which gives us this insight but predicts badly, I would choose the latter, and I am inclined to think that most economists would do the same." - Ronald Coase, Nobel-prize winning economist.

"I now take causal relationships to be the fundamental building blocks both of physical reality and of human understanding of that reality, and I regard probabilistic relationships as but the surface phenomena of the causal machinery that underlies and propels our understanding of the world". - Judea Pearl, Causality.

Imagination in Problem Creation "Perhaps no single phenomenon reflects the positive potential of human nature as much as intrinsic motivation, the inherent tendency to seek out novelty and challenges, to extend and exercise one's capacities, to explore, and to learn. Development lists acknowledge that from the time of birth, children, in their healthiest states, are active, inquisitive, curious, and playful, even in the absence of specific rewards" (Ryan and Deci 2000).

\section{Reputation in the Long-Run}

This paper reviews limitations of traditional control system and modern control system controllers, which are overcome to some extent using artificial intelligent techniques, such as ANN, Fuzzy Logic, Expert System, and Particle Swarm Optimization, Genetic Algorithm, etc. The review shows that efforts are made towards Power System Stabilizer based on Artificial Intelligent Techniques, which will give positive impact on the system stabilities and improve system performances.

\section{Applying Artificial Intelligence Techniques to Prevent Cyber Assaults}

Cyber security ostensibly is the discipline that could profit most from the introduction of Artificial Intelligence (AI). It is tough to make software for defending against the powerfully developing assaults in systems. It can be cured by applying techniques of artificial intelligence. Where conventional security systems may be slow and deficient, artificial intelligence techniques can enhance their overall security execution and give better security from an expanding number of complex cyber threats. Beside the great opportunities attributed to AI inside cyber security, its utilization has legitimized risks and concerns. To promote increment the development of cyber security, a holistic perspective of associations cyber environment is required in which $\mathrm{AI}$ is consolidated with human knowledge, since neither individuals nor AI alone has proven overall success in this sphere. In this manner, socially mindful utilization of AI techniques will be needed to further mitigate related risks and concerns

\section{Methodology}

\section{Artificial intelligence \& machine learning will improve trust}

Online marketplaces such as eBay, Taobao, Airbnb, and many others have grown dramatically since their inception just over two decades ago, providing businesses and individuals with previously unavailable opportunities to purchase or profit from online trading. Wholesalers and retailers can market their goods or get rid of excess inventory; consumers can easily search marketplaces for whatever is on their mind, alleviating the need for businesses to invest in their own e-commerce website; individuals transform items they no longer use into cash; and more recently, the so called "gig economy" is comprised of marketplaces that allow individuals to share their time or assets across different productive activities and earn extra income.

The amazing success of online marketplaces was not fully anticipated, primarily because of the hazards of anonymous trade and asymmetric information. Namely, how can strangers who have never transacted with one another, and who may be thousands of miles apart, be willing to trust each other? Trust on both 
sides of the market is essential for parties to be willing to transact and for a marketplace to succeed.

The scope for market design goes far beyond the more mainstream application like setting rules of bidding and reserve prices for auctions, or designing tiers of services, and in our view, includes the design of mechanisms that help foster trust in marketplaces. What follows are two examples from recent research that show some of the many ways that marketplaces can apply AI to the data they generate to help create more trust and better experiences for their customers.

\section{A. Using AI to assess the quality of sellers}

One of the ways that online marketplaces help participants build trust is by letting them communicate through online messaging platforms. For example, on eBay, buyers can contact sellers to ask them questions about their products, which may be particularly useful for used or unique products for which buyers may want to get more refined information than is listed.

Similarly, Airbnb allows potential renters to send messages to hosts and ask questions about the property that may not be answered in the original listing.

Using Natural Language Processing (NLP), a mature area in AI, marketplaces can mine the data generated by these messages in order to better predict the kind of features that customer's value. However, there may also be subtler ways to apply AI to manage the quality of marketplaces.

The messaging platforms are not restricted to pretransaction inquiries, but also offer the parties to send messages to each other after the transaction has been completed.

An obvious question then emerges: how could a marketplace analyze the messages sent between buyers and sellers post the transaction to infer something about the quality of the transaction that feedback doesn't seem to capture? This question was posed and answered in a recent paper by Masterov et al. (2015) using internal data from eBay's marketplace.

Buys a product and is given incentives to leave feedback, will leave positive feedback only if the buying experience was satisfactory. Hence, a seller will offer RFF incentives to buyers only if the seller expects to receive positive feedback, and this will happen only if the seller will provide high quality. If a seller knows that their goods and services are unsatisfactory, then paying for feedback will generate negative feedback that will harm the low-quality seller. Equilibrium behavior then implies that RFF, as a signal of high quality, will attract more buyers and result in more sales. The role of AI was precisely to reward buyers for information, not for positive feedback.

\section{STAGES}

In the first stage, the goal was to see if NLP can identify transactions that went bad when there was an independent indication that the buyer was unhappy. To do this, they collected internal data from transactions in which messages were sent from the buyer to the seller after the transaction was completed, and matched it with another internal data source that recorded actions by buyers indicating that the buyer had a poor experience with the transactions. Actions that indicate an unhappy buyer include a buyer claiming that the item was not received, or that the item was significantly not as described, or leaves negative or neutral feedback, to name a few.

The simple NLP approach they use creates a "poorexperience" indicator as the target (dependent variable) that the machine learning model will try to predict, and uses the messages' content as the independent variables. In its simplest form and as a proof of concept, a regular expression search was used that included a standard list of negative words such as "annoyed," "dissatisfied," "damaged," or "negative feedback" to identify a message as negative. If none of the designated terms appeared then the message was considered neutral. Using this classification, they grouped transactions into 3 distinct types: (1) No post-transaction messages from buyer to seller; (2) One or more negative messages; or (3) One or more neutral messages with no negative messages.

Figure 2, which appears in Masterov et al. (2016), describes the distribution of transactions with the different message classifications together with their association with poor experiences. The x-axis of Figure 1 shows that approximately $85 \%$ of transactions fall into the benign first category of no post-transaction messages. Buyers sent at least one message in the remaining $15 \%$ of all transactions, evenly split between negative and neutral messages. 
The top of the y-axis shows the poor experience rate for each message type. When no messages are exchanged, only $4 \%$ of buyers report a poor experience. Whenever a neutral message is sent, the rate of poor experiences jumps to $13 \%$, and if the message's content was negative, over a third of buyers express a poor experience.

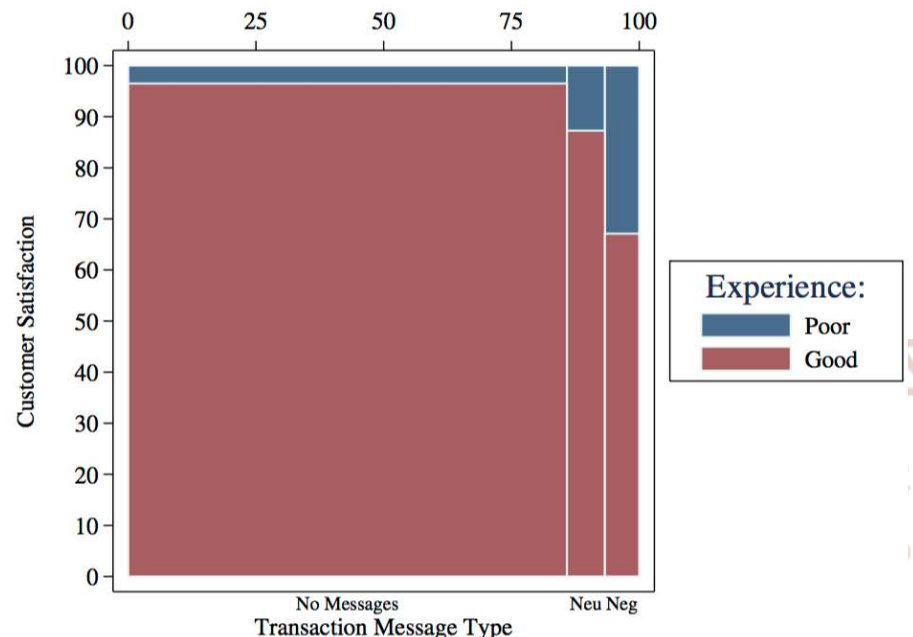

Figure 2: Message Content and Poor Experiences on eBay

In the second stage of the analysis, Masterov et al. (2016) used the fact that negative messages are associated with poor experiences to construct a novel measure of seller quality based on the idea that sellers who receive a higher frequency of negative messages are worse sellers. For example, imagine that seller A and seller B both sold 100 items and that seller A had five transactions with at least one negative message, while seller B there had eight such transactions. The implied quality score of seller $\mathrm{A}$ is then 0.05 while that of seller B is 0.08 and the premise is that seller B is a worse seller than seller A. Masterov et al. (2016) show that the relationship between this ratio, which is calculated for every seller at any point in time using aggregated negative messages from past sales, and the likelihood that a current transaction will result in a poor experience, is monotonically increasing.

This simple exercise is a proof of concept that shows that using the message data and a simple natural language processing AI procedure, they were able to better predict which sellers will create poor experiences than one can infer from the very inflated feedback data. eBay is not unique in allowing the parties to exchange messages and the lessons from this research are easily generalizable to other marketplaces. The key is that there is information in communication between market participants, and past communication can help identify and predict the sellers or products that will cause buyers poor experiences and negatively impact the overall trust in the marketplace.

\section{B. Using AI to create a market for feedback}

Aside from the fact that feedback is often inflated as described earlier, another problem with feedback is that not all buyers choose not to leave feedback at all. In fact, through the lens of mainstream economic theory, it is surprising that a significant fraction of online consumers leave feedback. After all, it is a selfless act that requires time, and it creates a classic free-rider problem. Furthermore, because potential buyers are attracted to buy from sellers, or products, that already have an established good track record, this creates a "cold start" problem: new sellers (or products) with no feedback will face a barrier-to-entry in that buyers will be hesitant to give them a fair shot. How could we solve these free-rider and cold-start problems?

These questions were analyzed in a recent paper by $\mathrm{Li}$ et al. (2017) using a unique and novel implementation of a market for feedback on the huge Chinese marketplace Taobao where they let sellers pay buyers to leave them feedback. Naturally, one may be concerned about allowing sellers to pay for feedback as it seems like a practice in which they will only pay for good feedback and suppress any bad feedback, which would not add any value in promoting trust. However, Taobao implemented a clever use of NLP to solve this problem: it is the platform, using an NLP AI model that decides whether feedback is relevant and not the seller who pays for the feedback. Hence, the reward to the buyer for leaving feedback was actually managed by the marketplace, and was handed out for informative feedback rather than for positive feedback.

Specifically, in March 2012, Taobao launched a "Rebate-for-Feedback" (RFF) feature through which sellers can set a rebate value for any item they sell (cash-back or store coupon) as a reward for a buyer's feedback. If a seller chooses this option then Taobao guarantees that the Taobao's motivation behind the RFF mechanism was to promote more informative feedback, but as Li et al. (2017) noted, economic theory offers some insights into how the RFF feature can act as a potent signaling mechanism that will further separate higher from lower quality sellers and products. To see this, recall the literature launched by Nelson (1970) who suggested that advertising acts as 
a signal of quality. According to the theory, advertising - this is a form of burning money - acts as a signal that attracts buyers who correctly believe that only high-quality sellers will choose to advertise. Incentive compatibility is achieved through repeat purchases: buyers who purchase and experience the products of advertisers will return in the future only if the goods sold are of high enough quality. The cost of advertising can be high enough to deter low quality sellers from being willing to spend the money and sell only once, because those sellers will not attract repeat customers, and still low enough to leave profits for higher quality sellers. Hence, ads act as signals that separate high quality sellers, and in turn attract buyers to their products.

As Li et al. (2017) argue, the RFF mechanism plays a similar signaling role as ads do. Assuming that consumers express their experiences truthfully in written feedback, any consumer who buys a product and is given incentives to leave feedback, will leave positive feedback only if the buying experience was satisfactory. Hence, a seller will offer RFF incentives to buyers only if the seller expects to receive positive feedback, and this will happen only if the seller will provide high quality. If a seller knows that their goods and services are unsatisfactory, then paying for feedback will generate negative feedback that will harm the low-quality seller. Equilibrium behavior then implies that RFF, as a signal of high quality, will attract more buyers and result in more sales. The role of AI was precisely to reward buyers for information, not for positive feedback.

Li et al. (2017) proceeded to analyze data from the period where the RFF mechanism was featured, and confirmed that first, as expected, more feedback was left in response to the incentives provided by the RFF feature. More importantly, the additional feedback did not exhibit any biases, suggesting that the NLP algorithms used were able to create the kind of screening needed to select informative feedback. Also, the predictions of the simple signaling story were borne out in the data, suggesting that using NLP to support a novel market for feedback did indeed solve both the free-rider problem and the cold-start problem that can hamper the growth of online marketplaces.

\section{B. Using AI to create a market for feedback}

Aside from the fact that feedback is often inflated as described earlier, another problem with feedback is that not all buyers choose not to leave feedback at all. In fact, through the lens of mainstream economic theory, it is surprising that a significant fraction of online consumers leave feedback. After all, it is a selfless act that requires time, and it creates a classic free-rider problem. Furthermore, because potential buyers are attracted to buy from sellers, or products, that already have an established good track record, this creates a "cold start" problem: new sellers (or products) with no feedback will face a barrier-to-entry in that buyers will be hesitant to give them a fair shot. How could we solve these free-rider and cold-start problems?

These questions were analyzed in a recent paper by $\mathrm{Li}$ et al. (2017) using a unique and novel implementation of a market for feedback on the huge Chinese marketplace Taobao where they let sellers pay buyers to leave them feedback. Naturally, one may be concerned about allowing sellers to pay for feedback as it seems like a practice in which they will only pay for good feedback and suppress any bad feedback, which would not add any value in promoting trust. However, Taobao implemented a clever use of NLP to solve this problem: it is the platform, using an NLP AI model that decides whether feedback is relevant and not the seller who pays for the feedback. Hence, the reward to the buyer for leaving feedback was actually managed by the marketplace, and was handed out for informative feedback rather than for positive feedback.

Specifically, in March 2012, Taobao launched a "Rebate-for-Feedback" (RFF) feature through which sellers can set a rebate value for any item they sell (cash-back or store coupon) as a reward for a buyer's feedback. If a seller chooses this option then Taobao guarantees that the rebate will be transferred from the seller's account to a buyer who leaves high-quality feedback. Importantly, feedback quality only depends on how informative it is, rather than whether the feedback is positive or negative. Taobao measures the quality of feedback with a NLP algorithm that examines the comment's content and length and finds out whether key features of the item are mentioned. Hence, the marketplace manages the market for feedback by forcing the seller to deposit at Taobao a certain amount for a chosen period, so that funds are guaranteed for buyers who meet the rebate criterion, which itself is determined by Taobao. 
Taobao's motivation behind the RFF mechanism was to promote more informative feedback, but as Li et al. (2017) noted, economic theory offers some insights into how the RFF feature can act as a potent signaling mechanism that will further separate higher from lower quality sellers and products. To see this, recall the literature launched by Nelson (1970) who suggested that advertising acts as a signal of quality. According to the theory, advertising - this is a form of burning money - acts as a signal that attracts buyers who correctly believe that only high-quality sellers will choose to advertise. Incentive compatibility is achieved through repeat purchases: buyers who purchase and experience the products of advertisers will return in the future only if the goods sold are of high enough quality. The cost of advertising can be high enough to deter low quality sellers from being willing to spend the money and sell only once, because those sellers will not attract repeat customers, and still low enough to leave profits for higher quality sellers. Hence, ads act as signals that separate high quality sellers, and in turn attract buyers to their products.

As Li et al. (2017) argue, the RFF mechanism plays a similar signaling role as ads do.

\section{CONCLUSION}

Classical economic theory paid little attention to market frictions, and treated information and computation as free. That theory led to conclusions about efficiency, competitive prices for most goods, and full employment of valuable resources. To address the failures of that theory, economists began to study model with search frictions, which predicted that price competition would be attenuated, that some workers and resources could remain unemployed, and that it could be costly to distinguish reliable trading partners from others. They also built markets for complex resource allocation problems in which computations and some communications were centralized, lifting the burden of coordination from individual market participants. With these as the key frictions in the traditional economy, AI holds enormous potential to improve efficiency. In this paper, we have described some of the ways that AI can overcome computational barriers, reduce search frictions, and distinguish reliable partners. These are among the most important causes of inefficiency in traditional economies, and there is no longer any question that $\mathrm{AI}$ is helping to overcome them, with the promise of widespread benefits for all of us.

\section{References}

1. Bughin, J., Hazan, E., Ramaswamy, S., Chui, M., Allas, T., Dahlstrom, P., Henke, N., and Trench, M. (2017) "Artificial Intelligence The Next Digital Frontier," McKinsey Global Institute Discussion Paper, June 2017.

2. Filippas, A., Horton, J. J. and Golden, J. M. (2017) "Reputation in the Long-Run," mimeo, NYU Stern School of Business.

3. Gal, M.S. and Elkin-Koren, N. (2017) "Algorithmic Consumers," Harvard Journal of Law \& Technology, 30:1-45.

4. Hutter, F., Hoos H., and Leyton-Brown, K. (2011) "Sequential Model-Based Optimization for General Algorithm Configuration,"

5. Leyton-Brown, K., Milgrom, P. R., and Segal, I. (2017) "Economics and Computer Science of a Radio Spectrum Reallocation," Proceedings of the National Academy of Sciences, 114:28,7202-7209. www.pnas.org/cgi/doi/10.1073/pnas.1701997114.

6. Li, L.I., Tadelis, S., and Zhou, X. (2016) "Buying Reputation as a Signal of Quality: Evidence from an Online Marketplace," NBER Working Paper No. 22584.

7. Masterov, D. V., Mayer, U. F., and Tadelis, S. (2015) "Canary in the e-commerce coal mine: Detecting and predicting poor experiences using buyer-to-seller messages," In Proceedings of the Sixteenth ACM Conference on Economics and Computation, EC'15, pp81-93.

8. Mayzlin, D., Dover, Y., and Chevalier, J. (2014) "Promotional reviews: An empirical investigation of online review manipulation," American Economic Review, 104(8):2421-55.

9. McCall, J.J. (1970) "Economics of information and job search," Quarterly Journal of Economics 84(1):113-126.

10. McSweeny, T. and O'dea, B. (2017) "The Implications of Algorithmic Pricing for Coordinated Effects, Analysis and Price Discrimination Markets in Antitrust Enforcement," Antitrust, 32(1):75-81.

11. Milgrom, P. R. (2017) Discovering Prices: Auction Design in Markets with Complex Constraints. Columbia University Press, 2017.

12. Milgrom, P. R., North, D.C. and Weingast, B. R. (1990) "The role of institutions in the revival of trade: The law merchant, private judges, and the Champagne fairs," Economics and Politics, 2(1):1-23. 24 